\title{
CLEANING OF VISCOUS DROPS ON A FLAT INCLINED SURFACE USING GRAVITY-DRIVEN FILM FLOWS
}

\author{
Julien R. Landel ${ }^{* 1}$, Harry McEvoy ${ }^{2}$ and Stuart B. Dalziel ${ }^{1}$ \\ ${ }^{1}$ Department of Applied Mathematics and Theoretical Physics, Centre for Mathematical \\ Sciences, Wilberforce Rd, Cambridge, CB3 OWA, UK. \\ ${ }^{2}$ Defence Science and Technology Laboratory, Porton Down, Salisbury, SP4 OJQ, UK.
}

\begin{abstract}
We investigate the fluid mechanics of cleaning viscous drops attached to a flat inclined surface using thin gravity-driven film flows. We focus on the case where the drop cannot be detached from the surface by the mechanical forces exerted by the cleaning fluid on the drop surface. The fluid in the drop dissolves into the cleaning film flow, which then transports it away. To assess the impact of the drop on the velocity of the cleaning fluid, we have developed a novel experimental technique based on particle image velocimetry. We show the velocity distribution at the film surface in the situations both where the film is flowing over a smooth surface, and where it is perturbed by a solid obstacle representing a very viscous drop. We find that at intermediate Reynolds numbers the acceleration of the starting film is overestimated by a plane model using the lubrication approximation. In the perturbed case, the streamwise velocity is strongly affected by the presence of the obstacle. The upstream propagation of the disturbance is limited, but the disturbance extends downstream for distances larger than 10 obstacle diameters. Laterally, we observe small disturbances in both the streamwise and lateral velocity, owing to stationary capillary waves. The flow also exhibits a complex three-dimensional converging pattern immediately below the obstacle.
\end{abstract}

KEYWORDS: cleaning surfaces; thin falling film; flow over obstacle; particle image velocimetry (PIV).

\section{INTRODUCTION}

Cleaning of fouling deposits using film flows is a common problem in many industrial processes, particularly in the food industry (see e.g. Wilson, 2004; Xin et al., 2002; Gillham et $a l .$, 2000). Fryer and Asteriadou (2009) explained that cleaning processes aim at overcoming cohesive forces within fouling deposits, as well as adhesive forces at the interface between a deposit and its substrate. Physical processes involve, for instance, fluid mechanical forces such as shear and pressure forces imposed at the deposit boundary by a liquid flow. Chemical processes can involve some material transport or diffusion and reactions. The shearing action of a film flow is often used to clean fouled surfaces in industrial processes as well as in our daily life (Yeckel and Middleman, 1987; Mickaily and Middleman, 1993), such as in a household dishwasher. In a full dishwasher, a jet of water impinges on the surface of some of the plates while others are simply covered by a thin draining film. The ability of the film to clean the drops of grease attached onto the plate surface is critical. Moreover, minimizing the water consumption and the energy of such automatic cleaning devices can have an important environmental and sustainable impact. Dussan (1987) analysed the effect of a shearing immiscible fluid flowing over a drop. He derived theoretically the rate of strain beyond which the drop is swept away by the fluid. In this study, we investigate the case where shear forces

\footnotetext{
*Corresponding author, email: J.Landel@damtp.cam.ac.uk
} 
cannot overcome adherence, and thus the drop remains attached onto the surface until it dissolves completely in the film.

We are interested in the case of cleaning a single drop of viscous liquid lying on an inclined planar surface using a gravity-driven falling film (see figure 1). Blount (2010) developed a mathematical model for the dissolution and transport of the fluid from the drop into the film flow. The streamwise velocity in the film is obtained assuming a viscous-gravity balance and the lubrication approximation,

$$
u_{\infty}(y)=\frac{g \sin \alpha}{2 v} y\left(2 h_{\infty}-y\right)
$$

where $y$ is the spatial coordinate in the direction orthogonal to the substrate ( $x$ the streamwise direction and $z$ the lateral or spanwise direction), $g$ is the constant of gravity, $\alpha$ is the inclination angle of the substrate from horizontal, $v$ is the film kinematic viscosity and $h_{\infty}$ is the far-field film thickness.

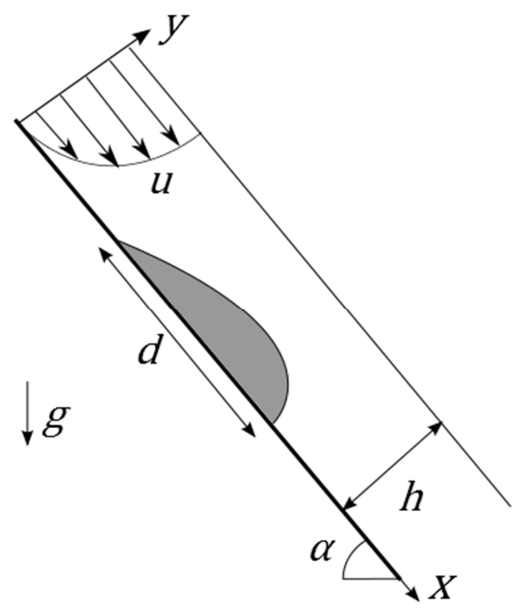

Figure 1. Schematic diagram of the cleaning problem. A liquid film flows over a viscous drop (shaded).

The drop fluid (shaded in figure 1), considered as a passive tracer, is described using the advection-diffusion equation in the film phase

$$
\partial_{t} A+\boldsymbol{u} \cdot \boldsymbol{\nabla} A=D \nabla^{2} A
$$

where $\partial_{t}$ is the partial differentiation with respect to time, $A$ is the local concentration of the drop fluid, $\boldsymbol{u}=(u, v, w)$ is the local film velocity and $D$ is the constant diffusion coefficient of the drop fluid in the film phase. Assuming that just outside the drop interface $A$ is fixed, and equal to the maximum solubility, $A_{s}$, of the drop fluid in the film phase, and that the film fluid forms a boundary layer such that $u \propto y$, Blount (2010) solved equation (2) to obtain a prediction for the total flux of drop fluid, integrated along the drop surface, into the film flow

$$
F=0.808 A_{S}\left(\frac{3 g^{2} D^{6} \Gamma d^{6} \sin ^{2} \alpha}{v^{2}}\right)^{1 / 9}
$$

where $\Gamma$ is the two-dimensional flow rate and $d$ is the drop length.

Our objective is to test the validity of the model developed by Blount (2010) and compare its prediction with experimental measurements. For simplicity, we focus here on the case of a 
non-deformable drop, which corresponds to the very viscous limit. One of the main assumptions in Blount's (2010) model is to consider that the film velocity is not affected by the drop and the velocity in the diffusive boundary layer remains linear with distance away from the boundary. To test this assumption we measure the velocity field of the film flow in the vicinity of a solid obstacle, representing a non-deformable drop.

The flow of a gravity-driven film falling along a rigid surface has been studied extensively (see e.g. the review of Craster and Matar, 2009). Since the pioneering work of Nusselt in 1916, who found a semi-parabolic velocity profile in the viscous-gravity regime, measuring the velocity field in film flows has always remained a technical challenge (see e.g. Moran et al. 2002, and Lel et al. 2005, for a review of various existing experimental techniques). Indeed, film flows are typically less than $1 \mathrm{~mm}$ thick and their velocity can reach $1 \mathrm{~m} \mathrm{~s}^{-1}$ at the free surface (Lan et al. 2010). Only recently, with the photochromic dye activation technique presented by Moran et al. (2002), the measurement of the instantaneous velocity field with a non-intrusive technique became possible. However, the measurements were still limited to only one location in the $(x, z)$ plane. Moreover, Moran et al.'s (2002) technique required the ability to see through the sides of the film, by confining the film inside a channel.

We have designed a new technique based on particle image velocimetry, which allows measurements of the two-dimensional instantaneous surface velocity of the film: i.e. $u(x, h, z, t)$ and $w(x, h, z, t)$ over an almost arbitrarily large area. This technique is nonintrusive, neglecting the impact of the tracer particles on the flow. It can achieve large temporal and spatial resolution, depending on the capabilities of the high-speed camera and the strength of the light source. It also enables us to measure the film surface velocity in the vicinity of obstacles, such as drops. The deformation of a liquid film flowing down an inclined plane due to obstacles has been extensively studied theoretically and numerically in the past 25 years. Pozrikidis \& Thoroddsen (1991) studied this problem theoretically and numerically for a three-dimensional film flowing over a particle, which is small compared to the film thickness. Using the boundary integral method for Stokes flow, Pozrikidis \& Thoroddsen (1991) built the Green's function to compute the perturbed flow field and the deformation of the interface. Based on this method, progress has been made recently in the theoretical and numerical modelling of the problem (see e.g. Hayes \& O'Brien, 2000; Gaskell et al., 2004; Blyth \& Pozrikidis, 2006; Baxter et al., 2009). However, owing to the difficulties mentioned above, there has been much less experimental work. To the best of our knowledge, there has not been any study reporting measurements of the velocity field in the vicinity of a film flowing over an obstacle. Experimentalists have usually focused on the measurement of the film thickness: the measurements of Decré \& Baret (2003) and Wierschem \& Askel (2004) compare well with the theoretical and numerical results.

Our goal in this work is to measure experimentally the influence of a non-deformable viscous drop on the velocity field of a film flowing over the drop. Ultimately, we want to characterize the impact on the dissolution and cleaning of drops lying over an inclined planar surface.

\section{EXPERIMENTAL PROCEDURES}

We produced gravity-driven thin film flows in the experimental apparatus shown schematically in figure 2. A liquid film flowed from a constant-head reservoir through a thin gap (thickness $h_{0}=0.4 \mathrm{~mm}$, width $L_{0}=200 \mathrm{~mm}$, length $l_{0}=15 \mathrm{~mm}$ ) on a flat solid substrate inclined at an angle $\alpha$ to the horizontal. We measured the angle of inclination $\alpha$ using an electronic inclinometer. At the gap outlet $(x=0)$, the flow could be well 
approximated by a plane Poiseuille flow. Then, the film flowed freely over a one millimetre thick sheet of polished stainless steel mounted on a $20 \mathrm{~mm}$ thick piece of PVC, which ensured the rigidity of the experimental apparatus. The stainless steel substrate was cleaned before each experiment with some water and soap, vinegar-based de-scaler and finally isopropanol. The film flowed on the substrate for a distance of approximately $300 \mathrm{~mm}$ from the outlet of the reservoir gap to the bottom-end of the substrate, and then fell freely into a large collecting tank. We obtained the flow rate of the film by measuring the flow rate of the pipe which supplied the reservoir and maintained the water level at a constant height. Using a precision balance (to measure the mass of fluid) and a stopwatch, the flow rate was measured and found to be consistent through repeated measurements with an accuracy of approximately $1 \%$. The fluid could recirculate in the experimental apparatus using a submersible pump located in the collecting tank. The fluid was pumped into a primary reservoir located upstream. The fluid turbulence in the primary reservoir was dampened as it penetrated through a piece of foam (reticulated polyether foam with 57 to 70 pores per inch and a pore size of $0.5 \mathrm{~mm}$ ) and a 5 $\mathrm{mm}$ gap into the main U-shaped reservoir. Using some artificial pearlescence (Iriodin 120 pigment, Merck) we observed that the fluid in the main U-shaped reservoir was free of turbulence.

Once the flow was stable and steady, we started recording the experiment with a high-speed grey-scale camera (Photron-Fastcam SA1.1) mounted with a $60 \mathrm{~mm}$ focal-length lens (AF Micro-Nikkor). Two 300 Watts arc lamps and two 250 Watts halogen lamps produced a uniform illumination on the film with minimal shadows or reflections at the crests and troughs of surface waves. The lens aperture was $\mathrm{f} / 4.0 \mathrm{D}$ or $\mathrm{f} / 5.6 \mathrm{D}$, depending on the shutter speed. The lens aperture was adjusted to prevent over-illumination. We recorded the experiments as 8-bit sequences with the high-speed camera for a duration of approximately $1 \mathrm{~s}$. We performed the PIV experiments in a dark room.

(a)

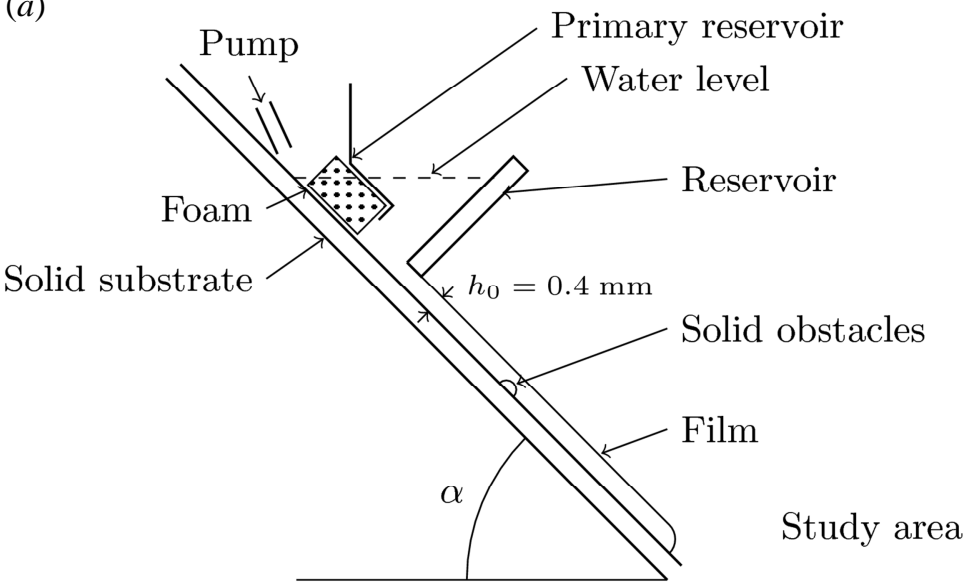

(b)

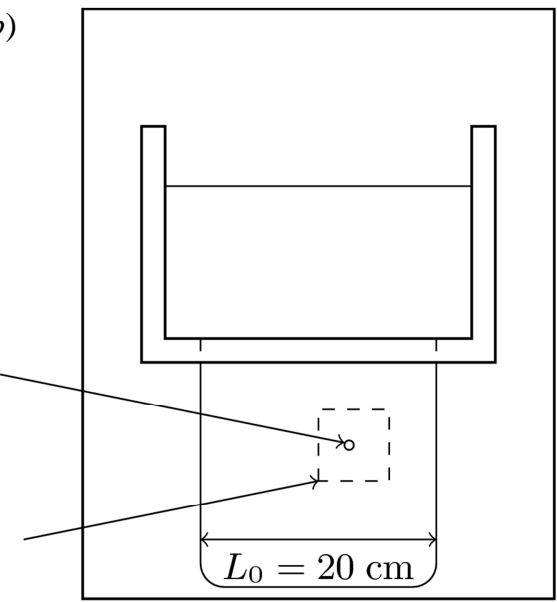

Figure 2. Schematic diagram of the experimental apparatus. (a) Side view; (b) top view.

The details of the control parameters for each experiment are presented in Table 1. For Exp. $1-4$, the camera view is centred on the film mid-width and with the top of the image just above the outlet, so as to see the film immediately after flowing through the gap. We analysed the images using DigiFlow (Dalziel et al., 2007). The spatial velocity resolution is $2.7 \mathrm{~mm}$ based on interrogation areas of $17 \times 17$ pixels with $75 \%$ overlapping. The film Reynolds number is defined as $\operatorname{Re}=4 \Gamma / v$ with $\Gamma(x)=Q / L(x)$ the local two-dimensional flow rate, $Q$ the three-dimensional constant flow rate, $L$ the local film width along the spanwise direction, 
and the viscosity of water $v=10^{-6} \mathrm{~m}^{2} \mathrm{~s}^{-1}$ at ambient temperature $T=20^{\circ} \mathrm{C}$. In Exp. 5, the camera view is centred on the obstacle in the flow. The resolution is approximately five times larger so that we have a very detailed measurement of the flow in the vicinity of the obstacle. The new spatial velocity resolution is $0.7 \mathrm{~mm}$ based on interrogation areas of $21 \times 21$ pixels with $5 \%$ overlapping.

The film liquid used for the PIV experiments was a mixture of approximately 4 litres of cold water with $40 \mathrm{~g}$ (i.e. $1 \% \mathrm{wt}$ ) of methylene blue and $20 \mathrm{~g}$ (i.e. $0.5 \% \mathrm{wt}$ ) of artificial pearlescence, which was made of titanium-dioxide coated mica particles (size: 5 to 25 microns; density: $3 \mathrm{~g} \mathrm{~cm}^{-3}$ ). The purpose of this very dark mixture of dye was to render the film opaque for the camera so that only the surface of the film could be seen. We noticed that methylene blue did not absorb strongly the infrared part of the spectrum of our lights. Since the camera was sensitive to this part of the spectrum, we mounted a UV/IR blocking filter on the camera lens to filter it out. The artificial pearlescence comprised small plates acting as tracers. Aligning with the shear, the plates predominantly orientated themselves parallel to the film surface. These tracers produced a non-uniform reflecting texture of light intensity at the surface of the film, from which the surface velocity could be computed using a PIV algorithm in DigiFlow. Since the particles of pearlescence were denser than water, they could sediment in the film flow. Assuming Stokes law for the settling velocity of the particles, we estimated that the smallest particles sank through less than $20 \%$ of the film depth. The sinking depth of the particle was of the order of the layer depth seen by the camera. Thus, there were always enough particles viewed by the camera for the PIV image analysis. To prevent sedimentation in the collecting tank a strong pump stirred the mixture vigorously.

Table 1. Summary of the control parameters for all the experiments.

\begin{tabular}{cccccccc}
\hline Exp. & $\begin{array}{c}\text { Angle } \\
\left({ }^{\circ}\right)\end{array}$ & $\begin{array}{c}Q \\
\left(\mathrm{~cm}^{3} \mathrm{~s}^{-1}\right)\end{array}$ & $\begin{array}{c}\text { Resolution } \\
(\text { pixel } \times \text { pixel })\end{array}$ & $\begin{array}{c}\text { View } \\
(\mathrm{cm} \times \mathrm{cm})\end{array}$ & $\begin{array}{c}\text { Frame } \\
\text { rate }(\mathrm{Hz})\end{array}$ & $\begin{array}{c}\text { Shutter } \\
\text { time }(\mathrm{s})\end{array}$ & $\begin{array}{c}\mathrm{Re}= \\
4 \Gamma / v\end{array}$ \\
\hline 1 & 44 & 50 & $1024 \times 1024$ & $17 \times 17$ & 2000 & $1 / 3000$ & $1000-1200$ \\
2 & 45 & 71 & $1024 \times 1024$ & $17 \times 17$ & 2000 & $1 / 2000$ & $1400-1700$ \\
3 & 45 & 41 & $1024 \times 1024$ & $17 \times 17$ & 2000 & $1 / 2000$ & $800-1000$ \\
4 & 61 & 65 & $1024 \times 1024$ & $15 \times 15$ & 2000 & $1 / 2000$ & $1300-1600$ \\
5 & 44 & 50 & $1024 \times 896$ & $3.4 \times 3.0$ & 6250 & $1 / 9000$ & 1100 \\
\hline
\end{tabular}

The impact of a solid obstacle on the film flow was studied. We made a small obstacle by sticking a piece of Blu-Tack (Bostik) on the substrate located at a distance of approximately $91 \mathrm{~mm}$ downstream of the outlet and approximately $10 \mathrm{~mm}$ to the right of the centreline. The size of the obstacle was 0.5 to $0.8 \mathrm{~mm}$ in thickness and $2.8 \mathrm{~mm}$ in diameter. The shape was a rough flattened hemisphere, which modelled the shape of a very viscous sessile drop. We can note that the obstacle was located sufficiently far away from the outlet that the film flow approaching the obstacle was fully developed. The obstacle was fully submerged by the film.

\section{RESULTS AND DISCUSSION}

In figure 3, we present the distribution of the local time-averaged surface velocity $\bar{u}$ of the film and its standard deviation (dashed curves) along the streamwise $(x)$ direction. These results correspond to Exp. 1 (see Table 1). The velocity is non-dimensionalised by the depthaveraged velocity as $x \rightarrow+\infty$, i.e. $\left\langle u_{\infty}\right\rangle$ computed from equation (1). The film Reynolds number varies from 1200 to 1000 , owing to the change of film width with streamwise distance. In figure $3(a)$, we plot with pluses the velocity distribution taken at a lateral $(z)$ location where the flow is undisturbed. We also plot with a thin solid line and a thick dashed 
line the theoretical fits for two values of $\beta=h_{0} / h_{\infty}$ (Cerro and Whitaker, 1971b). Cerro and Whitaker (1971b) modelled the laminar, steady, plane flow of a film falling on an inclined plane as it develops from a Poiseuille profile at the outlet $x=0$ (thickness $h_{0}$ ) to the viscousgravity profile described in (1) as $x \rightarrow+\infty$ (thickness $h_{\infty}$ ). They make use of the boundary layer approximation to simplify the Navier-Stokes equation, and then use the von Mises transformation (Schlichting, 1956, chap. 8) to account for the kinematic condition at the free surface. We solve the resulting non-linear partial differential equation, which also includes the continuity equation, using a finite-difference implicit numerical scheme.
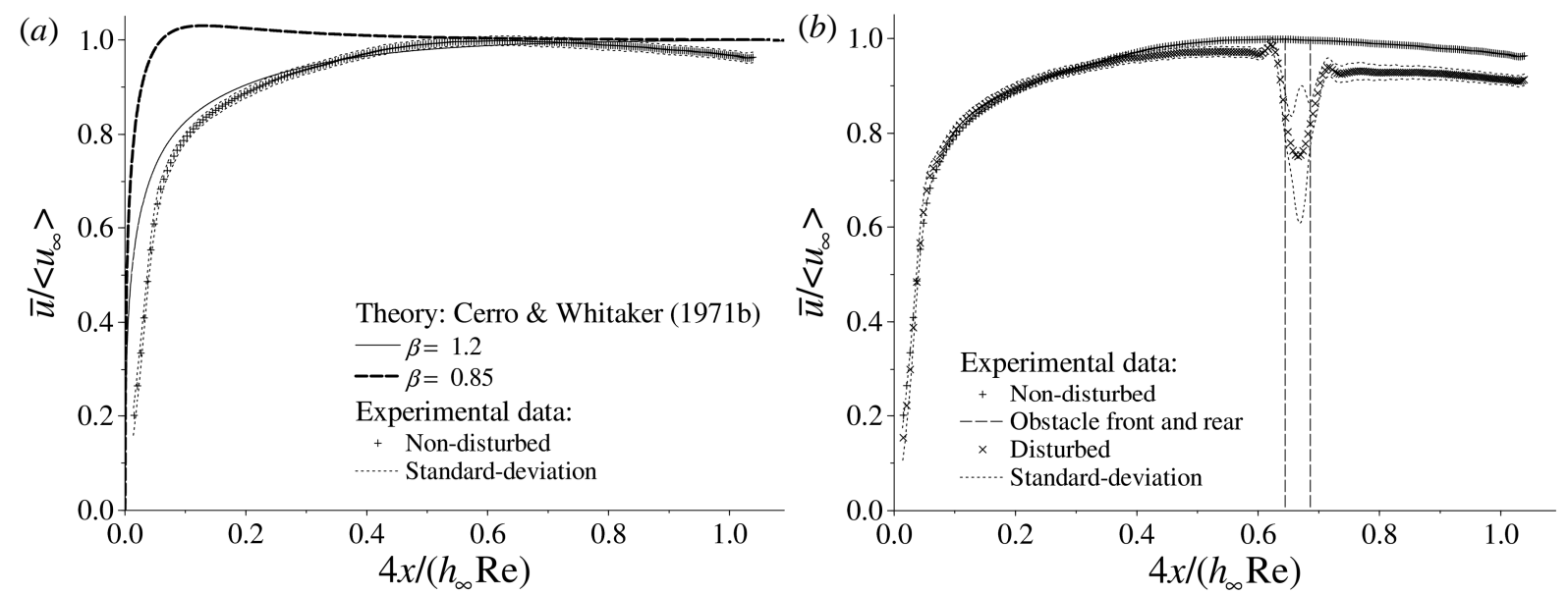

Figure 3. Experimental data from Exp. 1 and comparison with the theoretical predictions of Cerro \& Whitaker (1971b). (a) Non-dimensional distribution of the time-averaged surface velocity of the film (pluses) and its standard deviation (dotted curves) along the streamwise $(x)$ direction at a lateral $(z)$ location where the flow is undisturbed. We also plot with a thin solid line and a thick dashed line the theoretical fits for two values of $\beta=h_{0} / h_{\infty}$. The film Reynolds number varies from 1200 to 1000 , owing to the change of film width with streamwise distance. (b) Non-dimensional distribution of the time-averaged surface velocity of the film (crosses) and its standard deviation (dotted curves) along the streamwise $(x)$ direction at a lateral $(z)$ location where the flow is disturbed by an obstacle (located between the two dashed vertical lines). The non-disturbed data are also plotted with pluses for comparison.

The thick dashed curve in figure 3(a) corresponds to the theoretical prediction for the value $\beta=0.85 \pm 0.15$. This curve does not fit the experimental data well in the transition region for $0 \leq \xi=4 x /\left(h_{\infty} \mathrm{Re}\right) \leq 0.5$ : the model predicts a much more rapid growth and even an overshoot, since $\beta<1$ (Cerro and Whitaker, 1971b). Instead, the experimental data are better fitted with the value $\beta=1.2$ (thin solid curve), which predicts a slower more stable growth of the surface velocity (Cerro and Whitaker, 1971a). We observed a similar discrepancy between the theoretical value of $\beta$ and the best fit value for experiments at various flow rates or inclination angles. An ensemble average of Exp. 1-4, shows a best fit for $\beta 35 \%$ larger than the theoretical value. We believe that this mismatch could in part be explained by the experimental error on the gap height of the slit $h_{0}$ (owing to technical imperfection). Secondly, the film is not constrained laterally within a channel but flows freely on a planar surface. Surface tension across the film tends to pull the film inwards, narrowing it. The adjustment is particularly rapid at the beginning for the first 3 or $4 \mathrm{~cm}$, with the edges of the film forming an angle of up to $30^{\circ}$ to the $x$-axis. However, one should expect the film flow to accelerate as the film width reduces. Thirdly, surface tension effects, owing to surfactants in the film, could reduce the velocity at the free surface. Surface tension is not included in the 
theoretical model, which assumes no shear at the free surface. This could also explain the mismatch between the experimental results and the theoretical model of Cerro and Whitaker (1971b), which consistently predicts a larger velocity in the acceleration region.

In figure $3(b)$, we plot with crosses the velocity distribution taken at a lateral $(z)$ location where the flow is disturbed by an obstacle, which is located within the two dashed vertical lines. The profile plotted at the location of the obstacle (crosses) shows a clear and strong disturbance of the time-averaged surface velocity both upstream and downstream of the obstacle. The propagation of the disturbance propagates approximately one obstacle diameter upstream. At $\xi \approx 0.6$ we can note first a very small decrease of the velocity followed by a slight increase. Then the velocity drops sharply over the obstacle, by approximately 20 to $50 \%$, compared with the undisturbed velocity (also plotted in figure $3 b$ with pluses). The decrease is found consistently throughout the different experiments. The velocity increases again after the flow passes the centre of the obstacle. However, we can see that in the wake of the obstacle the surface velocity remains $5 \%$ lower than the undisturbed velocity. Comparing the different experiments, the velocity recovers its undisturbed value after 5 or more obstacle diameters downstream. The recovery distance tends to increase with Reynolds number. The profile of the disturbed surface velocity presented in figure $3(b)$ is typical across all the experiments. Only the magnitude of the velocity reduction and the recovery distance vary between the experiments. We believe that the velocity reduction is strongly related to the film thickness at the obstacle.
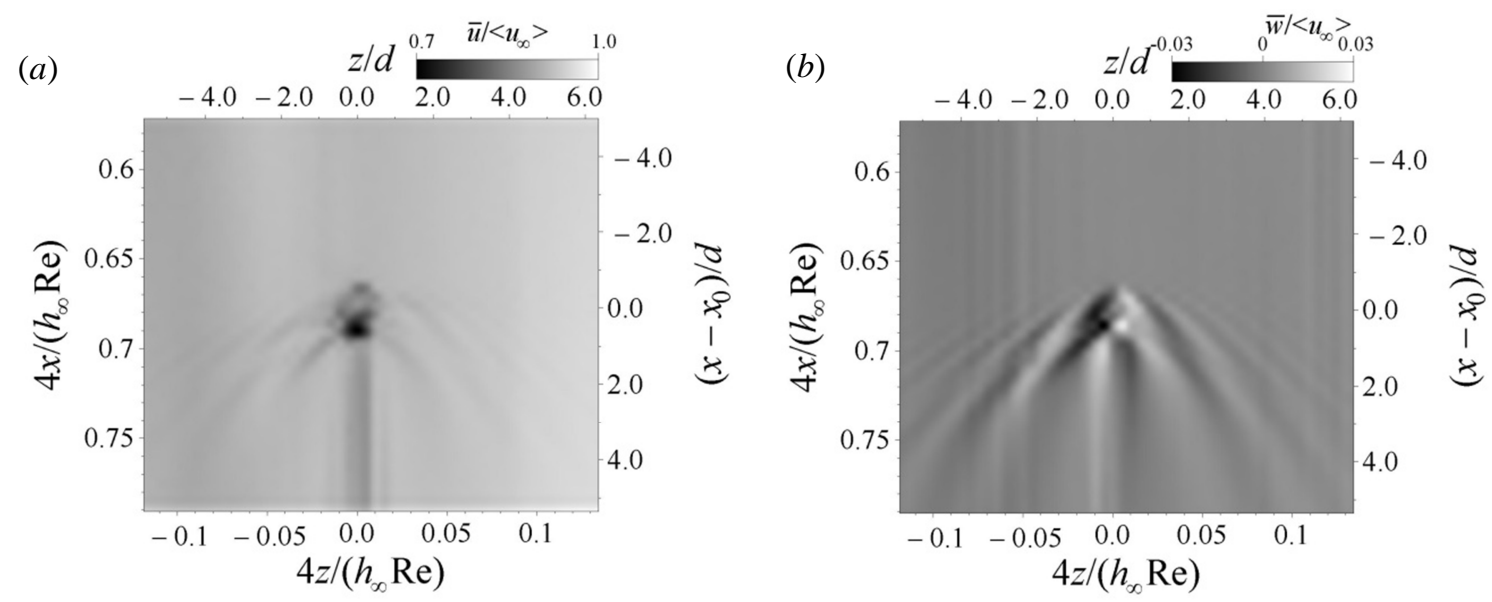

Figure 4. Spatial distribution of the surface velocity of a film flowing over a fully submerged obstacle (located at $(x, z)=\left(x_{0}, 0\right)$ on the right-hand-side and top axis) at an angle of $44^{\circ}$, a flow rate of $50 \mathrm{~cm}^{3} \mathrm{~s}^{-1}$ (Exp. 5 in Table 1). (a) Non-dimensional timeaveraged streamwise velocity; $(b)$ non-dimensional time-averaged lateral velocity (with negative values, in darker grey, pointing to the left).

In figure 4 , we show the spatial distribution of the surface velocity for the non-dimensional time-averaged streamwise velocity $\left.\bar{u} /<u_{\infty}\right\rangle$ (figure $4 a$ ), and the non-dimensional timeaveraged lateral velocity $\left.\bar{w} /<u_{\infty}\right\rangle$ (figure $\left.4 b\right)$. The obstacle is located at $(x, z)=\left(x_{0}, 0\right)$ on the right-hand-side and top axis. Upstream of the obstacle, we can see that the amplitude of the time-averaged surface velocities $\bar{u}$ and $\bar{w}$ are fairly uniform. In figure 4(a), we can notice a small and gradual increase, by 6 to $7 \%$ compared with the maximum amplitude, in the amplitude of the streamwise velocity from left to right. This variation could be due to a small misalignment between the camera and the substrate. Moreover, we can observe, in figure 4(b), 
vertical white bands in the lateral velocity field, denoting small variations of approximately $5 \%$ compared with the maximum amplitude. These bands could be due to a non-uniformity of the tracers, which we have observed to segregate into streaks downstream of very small defects. The defects could be lying on the substrate or at the gap outlet, such as: variations in surface roughness, or some solid particles or micro bubbles stuck on the surface.

In figure 4(a), we can see that the impact of the obstacle on the streamwise velocity is very limited upstream, but spreads laterally due to the formation of stationary capillary waves. These capillary waves, or 'bow waves', have a characteristic V shape similar to the wave front in the wake of ships. Pozrikidis and Thoroddsen (1991) observed that the amplitude of bow waves decrease in an exponential fashion with downstream distance away from the obstacle. Gaskell et al. (2004) noticed that the spread of the bow wave could be wellrepresented by an inverse hyperbolic cosine function. The reduction in the velocity is concentrated on the obstacle and also immediately downstream of the obstacle. As we observed in figure 3, the magnitude of the velocity does not recover its upstream value in the wake of the obstacle, for a band ranging the full width of the obstacle.

In figure 4(b), we should first note that the magnitude of the lateral velocity is at most $3 \%$ of the magnitude of the undisturbed streamwise velocity. The diverging flow on the obstacle is clearly visible in the velocity field, starting exactly at the top edge of the obstacle. Then, immediately downstream of the obstacle, $\bar{w}$ points inwards revealing flow convergence in a narrow region extending more than five obstacle diameters downstream. At the bottom edge of the obstacle, the flow is quite complex and three-dimensional. We find that the standard deviation is rather large in this region. It is possible that the tracers segregate away from this region owing to the divergence of the flow immediately upstream. Therefore, there might be less information for the computation of the velocity field, and the velocity field is slightly less accurate in a narrow band downstream of the obstacle. The V-shape pattern of the stationary capillary waves is also clearly revealed by the distribution of the lateral velocity.

\section{CONCLUSION}

We investigate the problem of cleaning a very viscous drop attached to an inclined surface by a gravity-driven falling film flowing over the drop. We are interested in the case where the film cannot detach the drop from the substrate. Instead, the drop fluid diffuses slowly into the cleaning film before being transported away by the bulk flow. This problem was modelled theoretically by Blount (2010) using an advection-diffusion equation. One of the key assumptions in the model is to consider that the drop does not impact the velocity in the diffusive boundary layer at the interface. To test this assumption, we have developed a new experimental technique, based on particle image velocimetry, to measure the velocity field at the surface of a liquid film. We report in this study the first measurements of the twodimensional distribution of the film surface velocity in the vicinity of an obstacle. The film Reynolds number is in the intermediate range: 800 to 1700. The flow is laminar, but inertial effects can be important.

First, we studied the undisturbed streamwise velocity profile, developing from a plane Poiseuille flow at the gap outlet, to the viscous-gravity regime in the far field. We found that the surface velocity reached asymptotically the viscous-gravity regime. However, the model developed by Cerro and Whitaker (1971b) consistently predicted a faster increase of the velocity. This discrepancy could be due to three-dimensional effects in the flow. The presence of surfactants and their impact on surface tension might also have had a strong influence on 
the surface velocity. As water flows out of the gap outlet of the main reservoir, the film free surface is created. The surfactants, which are naturally present in tap water, are gradually adsorbed by the free surface, as they rise from within the bulk of the fluid to the newly created free surface. Hence, a positive gradient in surfactant concentration can exist at the film surface along the streamwise direction. This gradient in surfactant concentration can lead to an opposite gradient in surface tension, thus decreasing the acceleration of the liquid at the free surface. Using a typical background concentration of surfactant in tap water of the order of $C_{b}=10^{-6} \mathrm{~mol} \mathrm{~m}^{-2}$ (per lateral unit length) and the relationship between surface tension and surfactant: $\gamma-\gamma_{b}=\mathrm{RT}\left(C_{b}-C\right)$ (where $\gamma$ is the surface tension, $\mathrm{R}$ is the ideal gas constant and the subscript $b$ indicates the background), we estimate a force gradient at the free surface acting opposite to the flow direction of the order of $10^{-3} \mathrm{~N} \mathrm{~m}^{-1}$. This result gives a reasonable estimate of the force needed to explain the mismatch between Cerro and Whitaker's (1971b) predictions of the film surface velocity and our measurements.

Second, we studied the impact of an obstacle on the film velocity. We observed a large decrease in the magnitude of the streamwise velocity starting one obstacle diameter upstream of the obstacle. The recovery of the streamwise velocity downstream of the obstacle can be larger than 10 obstacle diameters. Laterally, characteristic V-shaped capillary waves perturb the velocity field. The magnitude of the disturbance due to the waves is small compared with the disturbance at the obstacle. We can also observe a complex three-dimensional converging flow just below the obstacle. The reduction of the film velocity and the decrease of the film thickness in the vicinity of the obstacle can have an impact on the mass transfer between the drop and the film. If we assume that, similarly to the case of convective mass transfer in a Blasius boundary layer above a flat plate, the thickness of the diffusive boundary layer above the drop is related to the Schmidt number and the local Reynolds number such that $\delta_{C} \sim$ $x \mathrm{Sc}^{-1 / 3} \mathrm{Re}_{x}^{-1 / 2}$, then we can note that $\delta_{C}$ increases with decreasing Reynolds number. Hence, the diffusive boundary layer thickness increases with decreasing velocity in the film: for instance, a decrease of $50 \%$ in the velocity corresponds to an increase of $40 \%$ in $\delta_{C}$. Furthermore, increasing the diffusive boundary layer thickness tends to decrease the mass transfer at the interface, which means a lower cleaning rate of the droplets.

In conclusion, the drop has a strong impact on the film velocity. Therefore, the diffusion and advection of the drop fluid can be significantly affected as the film velocity decreases in the vicinity of the drop. The return flow immediately downstream of the drop could also have an effect of the cleaning process.

\section{ACKNOWLEDGEMENTS}

We wish to thank D. Page-Croft and the technicians of the GK Batchelor Laboratory at the Department of Applied Mathematics and Theoretical Physics, Cambridge. J. R. Landel wishes to thank his colleagues P. Luzzatto-Fegiz and F. Peaudecerf for fruitful discussions. J. R. Landel acknowledges financial support from Magdalene College, Cambridge, through a Nevile Research Fellowship in Applied Mathematics. This material is based upon work supported by the Defense Threat Reduction Agency under Contract No. HDTRA1-12-D0003-0001.

\section{REFERENCES}

Baxter, S. J., Power, H., Cliffe, K. A. and Hibberd, S. (2009) Three dimensional thin film flow over and around an obstacle on an inclined plane. Physics of Fluids 21, 32102. 
Blount, M. (2010) Aspects of advection-diffusion-reaction flows of relevance to decontamination. KTN Internship Report.

Blyth, M. G. and Pozrikidis, C. (2006) Film flow down an inclined plane over a threedimensional obstacle, Phys. Fluids 18, 052104.

Cerro, R. L. and Whitaker, S. (1971a) Stability of falling liquid films, Chem. Eng. Sc. 26, $742-745$

Cerro, R. L. and Whitaker, S. (1971b) Entrance region flows with a free surface: the falling liquid film, Chem. Eng. Sc. 26, 785-798.

Craster, R. V. and Matar, O. K. (2009) Dynamics and stability of thin liquid films, R. Mod. Phys. 18, 1131-1198.

Dalziel, S. B., Carr, M., Sveen, J. K. and Davies, P. A. (2007) Simultaneous synthetic schlieren and PIV measurements for internal solitary waves. Meas. Sc. Tech. 18, 533-547.

Decré, M. M. J. and Baret, J.-C. (2003) Gravity-driven flows of viscous liquids over twodimensional topographies, J. Fluid Mech. 487, 147-166.

Dussan V., E. B. (1987) On the ability of drops to stick to surfaces of solids. Part 3. The influences of the motion of the surrounding fluid on dislodging drops, J. Fluid Mech. 174, 381-397.

Gaskell, P. H., Jimack, P. K., Sellier, M., Thompson, H. M. and Wilson, M. C. T. (2004) Gravity-driven flow of continuous thin liquid films on non-porous substrates with topography, J. Fluid Mech. 509, 253-280.

Gillham, C. R., Fryer, P. J., Hasting, A. P. M. and Wilson, D. I. (2000) Enhanced cleaning of whey protein soils using pulsed flows, J. Food Eng. 46, 199-209.

Hayes, M. \& O'Brien, S. B. G. (2000) Green's function for steady flow over a small twodimensional topography. Physics of Fluids 12, 2845-2858.

Lan, H., Wegener, J. L., Armaly, B. F. and Drallmeier, J. A. (2010) Developing laminar gravity-driven thin liquid film flow down an inclined plane, J. Fluids Eng. 132, 081301.

Lel, V. V., Al-Sibai, F., Leefken, A. and Renz, U. (2005) Local thickness and wave velocity measurement of wavy films with a chromatic confocal imaging method and a fluorescence intensity technique, Exp. Fluids 39, 856-864.

Mickaily, E. S. and Middleman, S. (1993) Hydrodynamic cleaning of a viscous film from the inside of a long tube, AIChE J. 39, 885-893.

Moran, K., Inumaru, J. and Kawaji, M. (2002) Instantaneous hydrodynamics of a laminar wavy liquid film, Int. J. Multi. Flow 28, 731-755.

Nusselt, W. (1916) Die oberflachenkondensation des wasserdamphes, VDI-Zs 60, 541.

Pozrikidis, C. and Thoroddsen, S. T. (1991) The deformation of a liquid film flowing down an inclined plane wall over a small particle arrested on the wall, Phys. Fluids A 3, 2546-2558.

Wierschem, A. and Askel, N. (2004) Influence of inertia on eddies created in films creeping over strongly undulated substrates. Physics of Fluids 16, 4566-4574.

Wilson, D. I. (2004) Challenges in cleaning: recent developments and future prospects, Heat Trans. Eng. 26(1), 51-59.

Xin, H., Chen, X. D. and Özkan, N. (2002) Cleaning rate in the uniform cleaning stage for whey protein gel deposits, Food Bio. Proc. 80C, 240-246.

Schlichting, S. (1956) Boundary Layer Theory. McGraw-Hill, N.Y.

Yeckel, A. and Middleman, S. (1987) Removal of a viscous film from a rigid plane surface by an impinging liquid jet, Chem. Eng. Com. 50, 165-175. 